\title{
Scale-invariant drain current in nano-FETs
}

\author{
U. Wulfa and H. Richter ${ }^{b}$ \\ Brandenburgische Technische Universität Cottbus, Fakultät 1, Postfach 101344, 03013 Cottbus, Germany \\ ${ }^{a}$ wulf@physik.tu-cottbus.de, ${ }^{b}$ richter@ihp-microelectronics.com
}

Submitted September 4, 2009, accepted January 12, 2010

Keywords: Scaling, nano-transistors, quantum-transport

\begin{abstract}
Starting from a three-dimensional transport model in the Landauer-Büttiker formalism we derive a scale-invariant expression for the drain current in a nano-transistor. Apart from dimensionless external parameters representing temperature, gate-, and drain voltage the normalized drain current depends on two dimensionless transistor parameters which are the characteristic length $l$ and -width $w$ of the electron channel. The latter quantities are the physical length and -width of the channel in units of the scaling length $\lambda=\hbar\left(2 m^{*} \epsilon_{F}\right)^{-1 / 2}$. Here $\epsilon_{F}$ is the Fermi energy in the source contact and $m^{*}$ is the effective mass in the electron channel. In the limit of wide transistors and low temperatures we evaluate the scale-invariant $I_{D}-V_{D}$ characteristics as a function of the characteristic length. In the strong barrier regime, i. e. for $l \gtrsim 20$ long-channel behavior is found. At weaker barriers source-drain tunneling leads to increasingly significant deviations from the long-channel behavior. We compare with experimental results.
\end{abstract}

\section{Introduction}

The transistor density in integrated circuits doubles every two years (Moores law). Increased mobility in a short transistor channel leads to higher performance and less energy consumption. In technology, the reduction of the channel length is accompanied with the introduction of high$k$ gate dielectrics, metal gates, and strain engineering [1]. Currently alternative material systems and -geometries are under intense consideration as well [2] including carbon nanotube FETs [3], multi-gate FETs [4], nanowire transistors [5], Schottky barrier FETs [6], FETs with high- $\mu$ channel material (Ge [7]or III-V [8]) or transistors in hybrid-orientation technology [9].

As well-known, in conventional transistors with decreasing channel length the desired longchannel behavior of a transistor is degraded by short-channel effects [10, 11, 12]. One major source of these short-channel effects is the multi-dimensional nature of the electrostatic field which causes a reduction of the gate voltage control over a short electron channel. A second source is the advent of quantum transport. The most obvious quantum short-channel-effect is the formation of a source-drain tunneling regime below threshold gate voltage. In Ref. [13] it was found that in the source-drain tunneling regime the $I_{D}-V_{D}$-traces show a positive bending as opposed to the negative bending for classically allowed transport. The source-drain tunnelingand the classically allowed transport regime are separated by a close-to linear threshold characteristic. Such a behavior is found in numerous MOSFETs with channel lengths in the range of a few tens of nanometers (see for example Refs. [14, 15, 16, 17, 18, 19, 7]). While in early scaling 
concepts long-channel behavior could be assumed [20, 21] in the meantime two-dimensional effects in the electric field have been introduced in scaling [22, 23, 24, 25]. This paper is devoted to scaling properties of quantum transport.

For the modeling of quantum transport in nano-field effect transistors a number of advanced methods exist including non-equilibrium Green's function techniques [26, 27, 28, 29, 30], the Wigner function approach [31, 32, 33], atomistic scale approximation schemes [34, 35, 36, 37] or Monte Carlo-based techniques [38, 39, 40]. Naturally, these approaches rely to an essential extend on numerical methods. Therefore in many cases the scaling properties of nanotransistors have been extracted from simulations of concrete systems with given physical sizes $[41,42,43,44,45]$. As a complementary approach, there is a number of simpler one-dimensional effective models which lead to a more compact description (for a review see Ref. [46] and more recently Refs. $[47,48,49])$. Based on such a relatively simple approach a scale-invariant expression for the drain current was proposed recently [50]. Here the quantity $\lambda=\hbar / \sqrt{2 m^{*} \epsilon_{F}}$ arises as a natural scaling length for quantum transport where $\epsilon_{F}$ is the Fermi energy in the source contact and $m^{*}$ is the effective mass of the charge carriers. In this paper we show how the scale-invariant expression for the drain current can be derived. Furthermore, comparing with experimental results we study the quantum short channel effects as a function of the dimensionless characteristic length $l=L / \lambda$, where $L$ is the physical length of the transistor channel finding qualitative agreement.

\section{Theory}

Starting from a complete three-dimensional model of a nano-transistor we derive in the Appendix a simpler one-dimensional effective model (see Figs. 1 (a) and (b)). In the latter model the effective potential acting on the current carrying charges is given by $V^{\text {eff }}(y \leq 0)=0$, $V^{e f f}(0 \leq y \leq L)=1-V_{D} y / L$, and $V^{e f f}(y \geq L)=-V_{D}=-e U_{D}$, where $U_{D}$ is the applied drain voltage. After a proper normalization a scale-invariant expression for the drain current results which is given by

$$
i=\left(m-v_{G}\right) \int_{0}^{\infty} d \hat{\epsilon}\left[s\left(\hat{\epsilon}-\frac{m}{m-v_{G}}\right)-s\left(\hat{\epsilon}-\frac{m-v_{D}}{m-v_{G}}\right)\right] \tilde{T}\left[\hat{\epsilon} ; l^{2}\left(m-v_{G}\right), \frac{v_{D}}{m-v_{G}}\right] .
$$

In this expression the normalized supply function for a wide transistor reads

$$
s(\hat{\alpha})=\frac{1}{2 \sqrt{\pi}} w \sqrt{u} F_{-\frac{1}{2}}\left[\left(v_{G}-m\right) \frac{\hat{\alpha}}{u}\right]
$$

the effective current transmission is given by

$$
\tilde{T}\left[\hat{\epsilon} ; l^{2}\left(m-v_{G}\right), \frac{v_{D}}{m-v_{G}}\right]=\hat{k}_{D}(\hat{\epsilon})\left|\hat{t}^{S}\left(\hat{\epsilon} ; \beta, \hat{v}_{D}\right)\right|^{2}\left[\hat{k}_{S}(\hat{\epsilon})\right]^{-1}
$$

and the normalized chemical potential $m=\mu / \epsilon_{F}$ is

$$
m(u)=u X_{\frac{1}{2}}\left(\frac{4}{3 \sqrt{\pi}} u^{-3 / 2}\right) .
$$

Furthermore, $F_{-1 / 2}$ is the Fermi-Dirac integral $F_{j}(u)=\Gamma(j+1)^{-1} \int_{0}^{\infty} d v v^{j} /\left(1+e^{v-u}\right)$ of order $-1 / 2$ and $X_{\frac{1}{2}}$ is the inverse function of $F_{1 / 2}$. The normalized current $i=I / I_{0}$ with $I_{0}=2 e \epsilon_{F} / h$ is 
a)

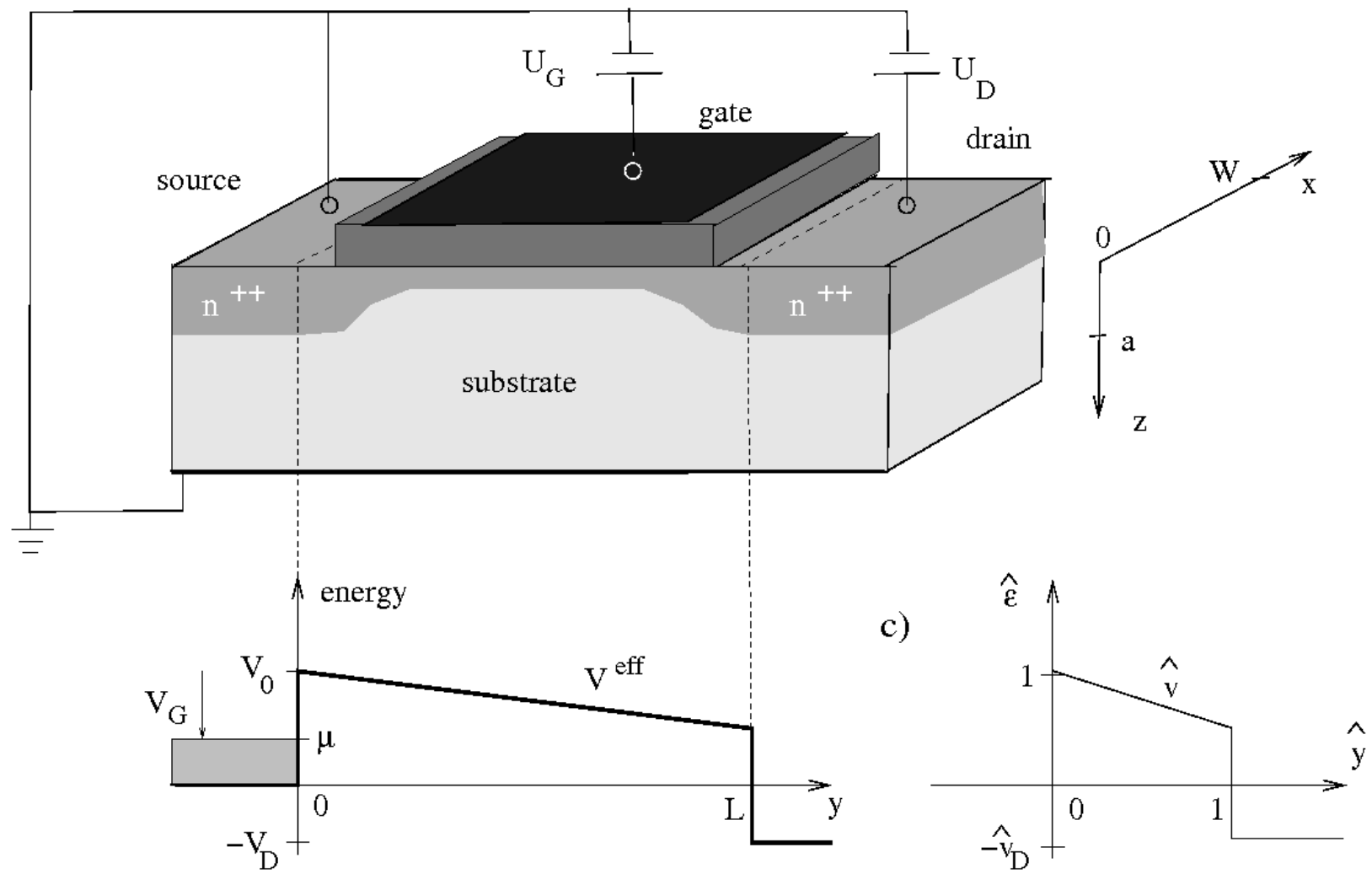

Fig. 1: a) Schematic representation of a generic n-channel nano-field effect transistor. b) Onedimensional effective potential $V^{e f f}$, and c) scaled effective potential $\hat{v}=V^{e f f} / V_{0}$ as a function of $\hat{y}=y / L$ entering Eq. 5 .

seen to depend on three normalized external parameters $v_{D}=V_{D} / \epsilon_{F}, v_{G}=V_{G} / \epsilon_{F}, u=k_{B} T / \epsilon_{F}$ and two dimensionless transistor parameters, namely the characteristic length $l=L / \lambda$ and width $w=W / \lambda, L$ and $W$ being the physical length and -width of the electron channel. As illustrated in Fig. 1 (b) the gate voltage parameter $V_{G}$ is defined as the deviation of the maximum of the source-drain barrier from the chemical potential in the source, $V_{G}=\mu-V_{0}$. The effective current transmission in Eq. 3 is calculated from the scattering solutions of the scaled one-dimensional Schrödinger equation

$$
\left[-\frac{1}{\beta} \frac{d^{2}}{d \hat{y}^{2}}+\hat{v}(\hat{y})-\hat{\epsilon}\right] \hat{\psi}(\hat{y}, \hat{\epsilon})=0,
$$

with $\beta=2 m^{*} V_{0} L^{2} / \hbar^{2}=l^{2}\left(m-v_{G}\right)$, and $\hat{y}=y / L$. The scaled effective potential $\hat{v}=V^{e f f} / V_{0}$ is given by $\hat{v}(\hat{y} \leq 0)=0, \hat{v}(0 \leq \hat{y} \leq 1)=1-\hat{v}_{D} \hat{y}$, and $\hat{v}(\hat{y} \geq 1)=-\hat{v}_{D}$, where $\hat{v}_{D}=$ $v_{D} /\left(m-v_{G}\right)$. As usual, the scattering functions emitted from source contact $\hat{\psi}^{S}$ obey the asymptotic conditions $\hat{\psi}^{S}(\hat{y} \leq 0, \hat{\epsilon})=\exp \left(i \hat{k}_{S} \hat{y}\right)+\hat{r}^{S}(\hat{\epsilon}) \exp \left(-i \hat{k}_{S} \hat{y}\right)$ and

$$
\hat{\psi}^{S}(\hat{y} \geq 1, \hat{\epsilon})=\hat{t}^{S}\left(\hat{\epsilon} ; \beta, \hat{v}_{D}\right) \exp \left(i \hat{k}_{D} \hat{y}\right)
$$

with $\hat{k}_{D}=\sqrt{\beta\left(\hat{\epsilon}+\hat{v}_{D}\right)}$ and $\hat{k}_{S}=\sqrt{\beta \hat{\epsilon}}$. The notation $\hat{t}^{S}\left(\hat{\epsilon} ; \beta, \hat{v}_{D}\right)$ is chosen to point out that because of Eq. 5 the scaled transmission coefficient only depends on the three parameters $\hat{\epsilon}, \beta$, and $\hat{v}_{D}$. 

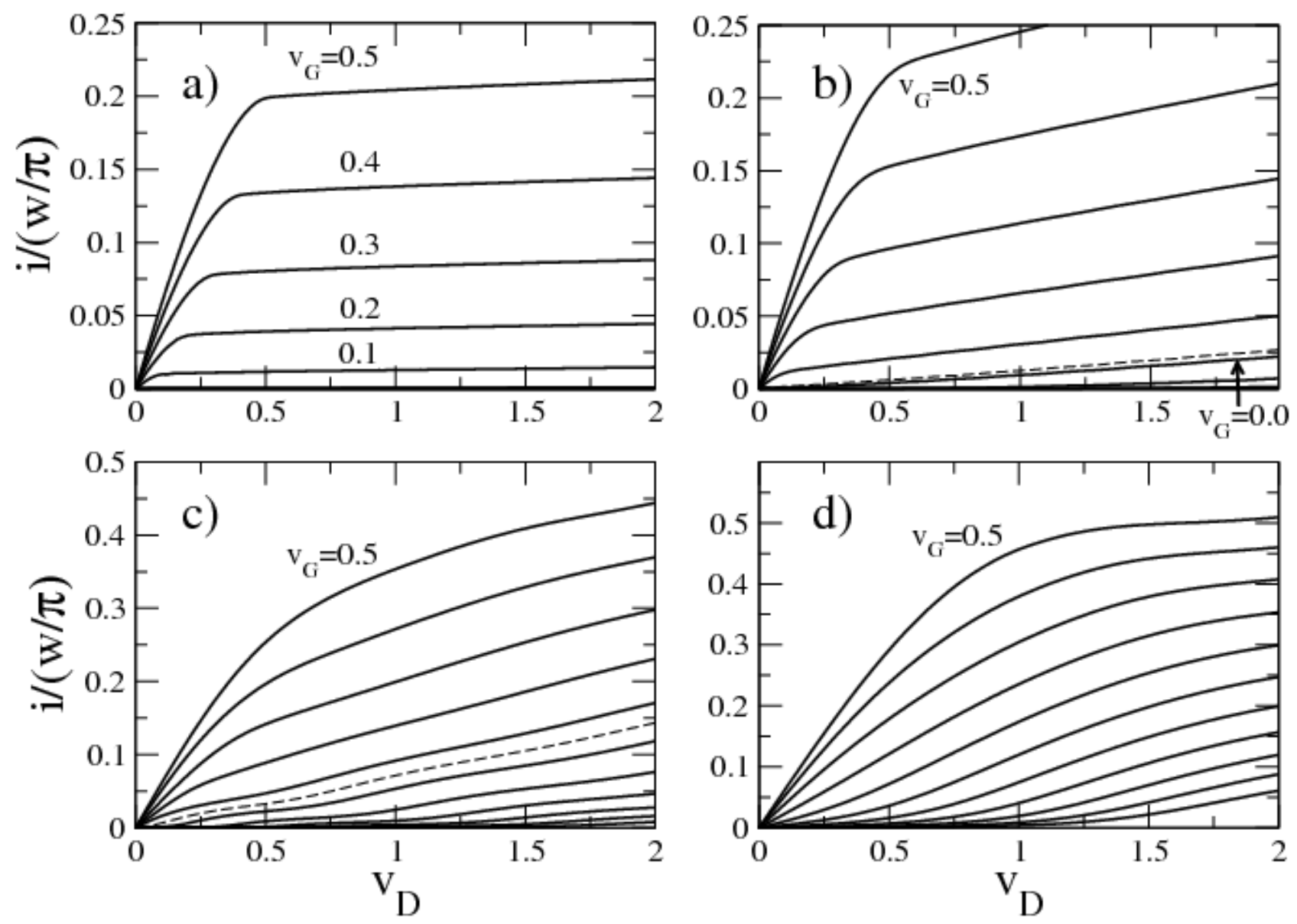

Fig. 2: Calculated low-temperature drain characteristics $(u=0.01)$ in the wide transistor limit, $v_{G}$ starting from 0.5 with decrements of 0.1 (solid lines). a) 'ideal limit' $l=500$, b) 'strong barrier' $l=40, \mathrm{c}$ ) 'transition regime' $l=10$, and d) 'weak barrier' $l=5$. In dashed lines $\mathrm{TH}$ (best fit to a linear characteristic) for $l=40$ at $v_{G}=0.02$ and for $l=10$ at $v_{G}=0.04$. For $l=500$ the $\mathrm{TH}$ is not resolvable from the abscissa and for $l=5$ it is absent.

\section{Numerical Results}

Evaluating Eq. 1 in the limit of low temperatures, $u \sim 0$, we analyze the dependence of $i / w$ on the three remaining dimensionless parameters $v_{G}, v_{D}$, and $l$. There are three possible types of plots, namely $i$ vs. $v_{D}$ at constant $v_{G}$ and $l$ ('drain characteristics' see Fig. 2), $i$ vs. $v_{G}$ at constant $v_{D}$ and $l$ ('transfer characteristics', Fig. $3(\mathrm{a})$ ), and $i$ vs. $l$ at constant $v_{D}$ and $v_{G}$ (Fig. $3(\mathrm{~b}))$.

From Figs. 2 (a) and 3 (a) it can be seen that Eq. 1 yields a typical long-channel behavior at very large $l$ ('ideal limit', $l=500$ in our numerical examples): First, the drain characteristics show for small drain voltages an approximately linear dependence of the drain current turning quite abruptly into a saturation regime for larger drain voltages. Second, the transfer characteristics demonstrate that the transistor can be blocked rapidly below threshold, exponentially fast.

In the strong barrier regime, represented by the calculation for $l=40$ in Fig. 2 (b), the drain characteristics still look similar to the long-channel characteristics. However, the traces 

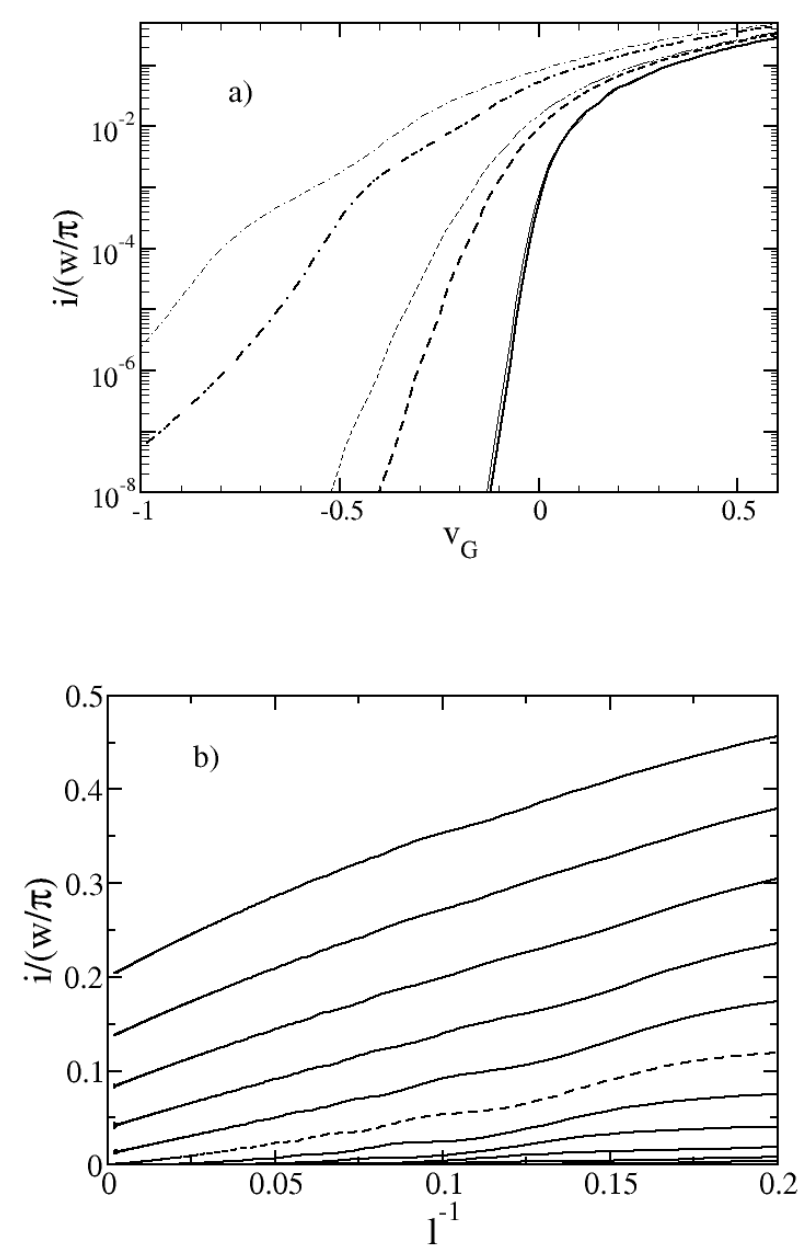

Fig. 3: a) Transfer characteristics for $l=500$ (solid lines), $l=40$ (dashed lines), and $l=10$ (dash-dotted lines). Fat lines at $v_{D}=1$, thin lines at $v_{D}=1.5$. b) Drain current versus characteristic length for $V_{D}=1.0$ and $v_{G}$ starting from 0.5 with decrements of 0.1 (dashed line $\left.v_{G}=0\right)$.

close to threshold gate voltage $v_{G} \sim 0$ are not given by $i \sim 0$. Instead, they can be described by a linear regression which increases in slope with decreasing characteristic length. This nearly linear regression has already been found in the three-dimensional transistor model in Ref. [51] where it was called close-to-linear threshold characteristic $(\mathrm{TH})$. Above the $\mathrm{TH}$, in the ONstate regime, the i-v traces are similar to the ones in the long-channel limit, however, instead of a current saturation only a quasi-saturation with a marked residual slope $d i / d v_{D}$ is found. Below the $\mathrm{TH}$, at $v_{G}<0$, the transistor works as a tunneling transistor. Here another type of characteristic arises showing a positive bending as opposed to the negative bending above the TH. Source-drain tunneling also causes a degradation of the transfer characteristics which at $l=40$ exhibit a weaker decrease of the drain current with decreasing negative $v_{G}$ (Fig. 3 (a)). In consequence for negative gate voltages only a quasi-OFF state is assumed. Furthermore a substantial dependence of the drain current on $v_{D}$ is found in the transfer characteristics. 

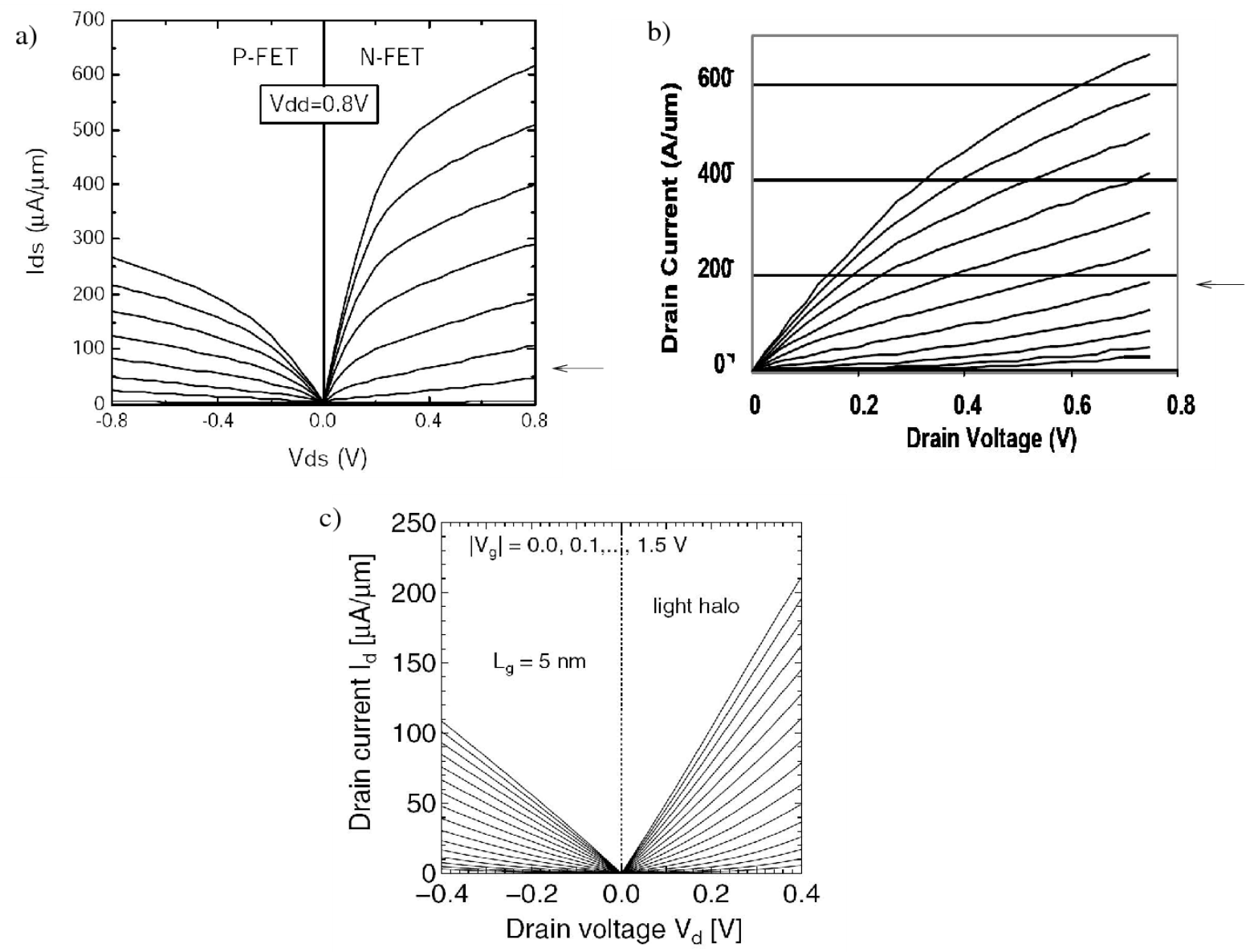

Fig. 4: Experimental drain characteristics: a) transistor with $15 \mathrm{~nm}$ gate length (see Fig. 3 of Ref. [14]), b) with 10nm gate length (see Fig. 13 of Ref. [16]), and c) with 5nm gate length (see Fig. 16 of Ref. [52]). We estimate the position of the TH as marked by an arrow.

In the transition regime to weak barriers $(l \sim 10$, see part $(c)$ of Fig. 2$)$ the traces for positive $v_{G}$ in the drain characteristics become more and more rounded so that it becomes difficult to define a quasi-saturation regime. In the quasi-OFF state regime small oscillations become noticeable in the i-v traces. In Ref. [13] it was demonstrated that these oscillations are due to resonant Fowler-Nordheim tunneling.

As shown in Fig. 2 (d) in the weak barrier limit, $l \lesssim 5$, the i-v traces differ qualitatively from the long-channel characteristics because of very strong source-drain tunneling. In particular no TH can be defined.

As in the drain characteristics the traces $i$ vs. $l^{-1}$ in Fig. 3 (b) develop a close-to-linear form at gate voltages close to zero. Above zero gate voltage the drain current tends to a finite value at large $l$ which is growing with $v_{G}$. At negative gate voltages the current vanishes for large $l$ increasingly fast with decreasing gate voltages.

\section{Discussion and conclusions}

We discuss our numerical results on the background of selected experimental drain characteristics reproduced in Fig. 4. To make contact with our theory we assume in absence of experimental 
values $\lambda=1 \mathrm{~nm}$ corresponding to a reasonable doping level of $N_{i} \sim 2 \times 10^{20} \mathrm{~cm}^{-3}$ (with Eq. 13 one obtains $\lambda=0.32 N_{V}^{1 / 3} N_{i}^{-1 / 3}$, where we take a valley degeneracy of $N_{V}=6$ ). At $\lambda=1 \mathrm{~nm}$ the experimental gate lengths of $L=15 \mathrm{~nm}, 10 \mathrm{~nm}$, and $5 \mathrm{~nm}$ correspond to characteristic lengths of $l=15,10$, and 5. Furthermore, the Fermi energy $\epsilon_{F} \sim 0.2 \mathrm{eV}$ is found from $\lambda=\hbar\left(2 m^{*} \epsilon_{F}\right)^{-1 / 2}$ with $\lambda=1 \mathrm{~nm}$ and $m^{*}=0.2 m_{0}$. Then the maximum of $v_{D}=2$ in our numerical calculations corresponds to an experimental drain voltage of $2 \epsilon_{F}=0.4 \mathrm{~V}$. Since in the experiments the series resistance of the source- and the drain contact has to be taken into account it can be expected that $v_{D}=2$ in fact corresponds to an experimental drain voltage between $0.4 \mathrm{~V}$ and $0.8 \mathrm{~V}$ which are the maximum experimental values considered in Fig. 4. With these presuppositions it is seen that the transistor with 10nm gate length in Fig. 4 (b) can be regarded to represent the transition regime of characteristic lengths around $l \sim 10$ with rounded $\mathrm{i}-\mathrm{v}$ traces (compare with Fig. 2 (c)). Likewise, the transistor with 15nm gate length in Fig. 4 (a) represents the lower edge of the strong barrier regime which is characterized by a marked transition between the initial linear- and the quasi-saturation regime (compare with Fig. 2 (b)). Finally, the 5nm gate length transistor in Fig. 4 (c) can be associated with the weak barrier regime with no TH and no quasi-saturation (see Fig. 2 (d)).

To conclude, we present a scaling-approach for quantum-transport in nano-transistors. In a simple model we find a scaling length for quantum transport and an expression for the dimensionless drain current as a function of five dimensionless parameters. These parameters are the normalized drain voltage, -gate voltage and -temperature as well as the characteristic length and -width of the electron channel. For a wide transistor and low temperatures we evaluate the drain characteristics numerically and find qualitative agreement with experiments.

\section{Appendix: The scaled one-dimensional effective model in detail}

Initial three-dimensional model Our starting point is the three-dimensional time-independent Schrödinger equation

$$
\left[-\frac{\hbar^{2}}{2 m^{*}} \Delta+V(\mathbf{r})-E\right] \Psi(\mathbf{r}, E)=0 .
$$

It is assumed that the potential acting on the current carrying charges is independent of the width-direction, $V(\mathbf{r})=V(y, z)$. The finite width of the transistor is represented by Dirichlet boundary conditions $\Psi(x=0, y, z, E)=\Psi(x=W, y, z, E)=0$. Writing

$$
\Psi(\mathbf{r}, E)=\psi(y, z, \epsilon) \sqrt{\frac{2}{W}} \sin \left(\frac{N_{x} \pi}{W} x\right)
$$

we obtain

$$
\left[-\frac{\hbar^{2}}{2 m^{*}}\left(\frac{\partial^{2}}{\partial y^{2}}+\frac{\partial^{2}}{\partial z^{2}}\right)+V(y, z)-\epsilon\right] \psi(y, z, \epsilon)=0
$$

where the total energy for the motion in the $y-z$-plane is $\epsilon=E-N_{x}^{2} E_{x}^{0}, E_{x}^{0}=\left(\hbar^{2} / 2 m^{*}\right)(\pi / W)^{2}$. In our scattering theoretical approach we furthermore assume for the source contact $V(z, y \leq$ $0)=v(z)$ and for the drain contact we set $V(z, y \geq L) \equiv v(z)-V_{D}$. Then in the contacts transversal modes $\Phi_{N}$ can be defined as the solutions of the eigenvalue problem

$$
\left[-\frac{\hbar^{2}}{2 m^{*}} \frac{d^{2}}{d z^{2}}+v(z)-E_{z}^{N}\right] \Phi_{N}(z)=0 .
$$


We neglect leakage currents to the top- and to the back gate setting $\Phi_{N}(0)=\Phi_{N}\left(z_{c}\right)=0$, where $z_{c}$ is a suitable cut-off parameter. To keep the presentation as simple as possible the free additive constant for $V(\mathbf{r})$ in Eq. 7 is chosen so that $E_{z}^{0}=0$. The scattering solutions $\psi^{S N}$ and $\psi^{D N}$ of Eq. 9 are defined by their asymptotic behavior in the contacts. For the scattering function $\psi^{S N}$ emitted from the source contact with a transversal mode $\Phi_{N}$ we write

$$
\psi^{S N}(y \leq 0, z, \epsilon)=\Phi_{N}(z) \frac{e^{i k_{S N} y}}{\sqrt{2 \pi}}+\sum_{N^{\prime}} r^{S ; N^{\prime} N} \Phi_{N^{\prime}}(z) \frac{e^{-i k_{S N^{\prime}} y}}{\sqrt{2 \pi}}
$$

and in the drain contact

$$
\psi^{S N}(y \geq L, z, \epsilon)=\sum_{N^{\prime}} t^{S ; N^{\prime} N} \Phi_{N^{\prime}}(z) \frac{e^{i k_{D N^{\prime}}(y-L)}}{\sqrt{2 \pi}} .
$$

Here the reflection- and transmission coefficients are given by $r^{S ; N^{\prime} N}$ and $t^{S ; N^{\prime} N}$, respectively, and the wave numbers by $k_{S N}=\sqrt{2 m^{*}\left(\epsilon-E_{z}^{N}\right)} / \hbar$ as well as $k_{D N^{\prime}}=\sqrt{2 m^{*}\left(\epsilon-E_{z}^{N^{\prime}}+V_{D}\right)} / \hbar$.

For a wide enough transistor and a sufficient junction depth $a$ (see Fig. 1) the electrons in the contacts can be treated as a three-dimensional non-interacting electron gas. The electron density in this case is given by $n_{0}=N_{V} N_{3 D} F_{1 / 2}\left[\mu /\left(k_{B} T\right)\right]$, where $N_{3 D}=2\left(2 \pi m^{*} k_{B} T / h^{2}\right)^{3 / 2}$ (see Sect. 1.5 of Ref. [46]) and $N_{V}$ is the valley-degeneracy factor. We assume that all impurities of density $N_{i}$ are ionized, independent of the temperature. In the zero temperature limit one has $F_{1 / 2}\left[\mu /\left(k_{B} T\right)\right] \rightarrow 4\left(\epsilon_{F} / k_{B} T\right)^{3 / 2} /(4 \sqrt{\pi})$ and thus

$$
n_{0}=N_{i}=N_{V} \frac{8}{3 \sqrt{\pi}}\left(\frac{2 \pi m^{*} \epsilon_{F}}{h^{2}}\right)^{3 / 2} .
$$

Equating the finite temperature expression for $n_{0}$ with the zero-temperature limit it results that

$$
\frac{\mu}{k_{B} T}=X_{1 / 2}\left[\frac{4}{3 \sqrt{\pi}}\left(\frac{\epsilon_{F}}{k_{B} T}\right)^{3 / 2}\right] .
$$

Drain current in the three-dimensional model From Eqs. 8, 11, and 12 it follows that the source-emitted scattering solutions of (7) are

$$
\Psi(\mathbf{r}, E)=\Psi^{S N N_{x}}(\mathbf{r}, \epsilon)=\psi^{S N}(y, z, \epsilon) \sqrt{\frac{2}{W}} \sin \left(\frac{\pi N_{x}}{W} x\right) .
$$

A transmitted component of $\Psi^{S N N_{x}}(\mathbf{r}, \epsilon)$ associated with the index $N^{\prime}$ in Eq. 12 only contributes to the current if the incident channel is open (real $k_{S N}$ ) as well as the transmitted one (real $\left.k_{D N^{\prime}}\right)$. For the current carried by such a state one obtains

$$
\begin{aligned}
& \int_{0}^{z_{c}} d z \int_{0}^{W} d x e j_{y}(\mathbf{r})=e \int_{0}^{z_{c}} d z \int_{0}^{W} d x \frac{\hbar}{m^{*}} \operatorname{Im}\left[\Psi^{S N N_{x}}(\mathbf{r}, \epsilon)^{*} \frac{\partial}{\partial y} \Psi^{S N N_{x}}(\mathbf{r}, \epsilon)\right] \\
= & \frac{e \hbar}{2 \pi m^{*}} \sum_{N^{\prime}}\left|t^{S N^{\prime} N}\right|^{2} k_{D N^{\prime}} \Theta\left(\epsilon-E_{z}^{N}\right) \Theta\left(\epsilon-E_{z}^{N^{\prime}}+V_{D}\right) \equiv I^{S N}(\epsilon) .
\end{aligned}
$$


The total current from source to drain, $I_{S \rightarrow D}$, results from summing over all scattering states $\Psi^{S N N_{x}}(\mathbf{r}, \epsilon)$ with a weight given by the Fermi distribution,

$$
I_{S \rightarrow D}=2 \sum_{N_{x}, N} \int_{0}^{\infty} d \epsilon g_{S N}(\epsilon) f\left(\epsilon+N_{x}^{2} E_{x}^{0}-\mu\right) I^{S N}(\epsilon) .
$$

The function $g_{S N}(\epsilon)=d k_{S N} / d \epsilon=m^{*} /\left[\hbar^{2} k_{S N}\right]$ is the one-dimensional density of states and $f(x)=\left[e^{x /\left(k_{B} T\right)}+1\right]^{-1}$. Since $E_{z}^{0} \equiv 0$ the Theta-functions in $I^{S N}(\epsilon)$ ensure that the energy integral ranges from zero to plus infinity. The factor of two in front of the r. h. s. of (17) accounts for the spin degeneracy. Formally the $N_{x}$-summation in (17) can be carried out defining the supply function

$$
S\left(\epsilon-\mu, E_{x}^{0}\right)=\sum_{N_{x}=1}^{\infty} f\left(\epsilon-\mu+N_{x}^{2} E_{x}^{0}\right) .
$$

Here, essentially a Riemann sum is obtained in the variable $N_{x} \sqrt{E_{x}^{0}}$ which in the limit of wide transistors, $E_{x}^{0} \rightarrow 0$, can be replaced by an integral yielding

$$
\lim _{E_{x}^{0} \rightarrow 0} S\left(\epsilon-\mu, E_{x}^{0}\right) \equiv S(\epsilon-\mu)=\sqrt{\frac{m^{*} k_{B} T}{2 \pi \hbar^{2}}} W F_{-1 / 2}\left(\frac{\epsilon-\mu}{k_{B} T}\right)
$$

Inserting Eqs. 19 and 16 in Eq. 17 as well as the expression for $g_{S N}$ results in

$$
I_{S \rightarrow D}=\frac{2 e}{h} \int_{0}^{\infty} d \epsilon S(\epsilon-\mu) \tilde{T}_{S \rightarrow D}(\epsilon),
$$

with

$$
\tilde{T}_{S \rightarrow D}(\epsilon)=\sum_{N, N^{\prime}} \Theta\left(\epsilon-E_{z}^{N}\right) \Theta\left(\epsilon-E_{z}^{N^{\prime}}+V_{D}\right) k_{D N^{\prime}}\left|t^{S N^{\prime} N}(\epsilon)\right|^{2} k_{S N}^{-1}
$$

Taking into account that the chemical potential in the drain contact is given by $\mu-V_{D}$ an analogous expression can be derived for the total current from drain contact to source contact

$$
I_{D \rightarrow S}=\frac{2 e}{h} \int_{0}^{\infty} d \epsilon S\left(\epsilon-\mu+V_{D}\right) \tilde{T}_{D \rightarrow S}(\epsilon) .
$$

As shown in Refs. [51, 13] (see in particular Eqs. A14 and A15 in the latter reference) it holds that $\tilde{T}_{S \rightarrow D}=\tilde{T}_{D \rightarrow S}=\tilde{T}_{S D}$. Then, for the total drain current $I=I_{S \rightarrow D}-I_{D \rightarrow S}$ the Eqs. 20 and 22 yield

$$
I=\frac{2 e}{h} \int_{0}^{\infty} d \epsilon\left[S(\epsilon-\mu)-S\left(\epsilon-\mu+V_{D}\right)\right] \tilde{T}_{S D}(\epsilon) .
$$


Effective Transmission The summations over $N$ and $N^{\prime}$ in Eq. 21 to obtain the total current transmission $\tilde{T}_{S D}$ in (23) represent intricate mode conversion processes between the incident and the transmitted part of the scattering functions. As demonstrated in Ref. [13] it is possible to introduce systematic approximations to derive a simpler one-dimensional effective scattering problem with

$$
\tilde{T}_{S D} \sim \tilde{T}^{e f f} .
$$

Here the effective current transmission $\tilde{T}^{e f f}=k_{D}^{e f f}\left|t^{S}\right|^{2}\left(k_{S}^{e f f}\right)^{-1}$ is calculated from the scattering solutions of

$$
\left[-\frac{\hbar^{2}}{2 m^{*}} \frac{d^{2}}{d y^{2}}+V^{e f f}(y)-\epsilon\right] \psi^{e f f}(y, \epsilon)=0
$$

with the effective potential $V^{\text {eff }}$ obeying the boundary conditions $V^{\text {eff }}(y<0)=0$ and $V^{e f f}(y>L)=-V_{D}$. The scattering solutions $\psi^{e f f ; S}$ emitted from the source contact obey the asymptotic relations $\psi^{\text {eff; } S}(y \leq 0, \epsilon)=\exp \left(i k_{S}^{\text {eff }} y\right)+r^{S} \exp \left(-i k_{S}^{\text {eff }} y\right)$ and

$$
\psi^{e f f ; S}(y \geq L, \epsilon)=t^{S}(\epsilon) e^{i k_{D}^{e f f} y}
$$

where $k_{S}^{e f f}=\sqrt{2 m^{*} \epsilon / \hbar^{2}}$ and $k_{D}^{e f f}=\sqrt{2 m^{*}\left(\epsilon+V_{D}\right) / \hbar^{2}}$. We list the requirements for (24) to be valid. The first requirement is a narrow confinement in the $\mathrm{z}$-coordinate so that it is appropriate to assume that the potential in the scattering area is separable,

$$
V(z, 0 \leq y L)=V_{z}(z)+V_{y}(y)
$$

The conclusions drawn in the separable potential can be generalized to the non-separable case as described in Ref. [53]. Second, because of the strong confinement in the z-direction only the lowest quantum level $E_{k=1} \equiv E_{1}$ in the electron channel has to be taken into account which is defined through the eigenvalue problem

$$
\left[-\frac{\hbar^{2}}{2 m^{*}} \frac{\partial^{2}}{\partial x^{2}}+V_{z}(z)-E_{k}\right] \phi_{k}(x)=0 .
$$

Third, the wave numbers $k_{S N}$ and $k_{D N}$ in can be replaced by effective ones setting in Eq. 21

$$
k_{S N} \sim k_{S}^{e f f}=\sqrt{2 m^{*} \epsilon / \hbar^{2}}, \quad \text { and } \quad k_{D N} \sim k_{D}^{e f f}=\sqrt{2 m^{*}\left(\epsilon+V_{D}\right) / \hbar^{2}} .
$$

Finally, evanescent modes (imaginary $k_{S N}$ or $k_{D N}$ ) can be neglected. Under these conditions one obtains the effective potential

$$
V^{e f f}(y)= \begin{cases}0 & \text { for } y<0 \\ E_{1}+V_{y}(y), & \text { for } 0 \leq L \\ -V_{D}, & \text { for } y>L\end{cases}
$$

to be entered in Eq. 25. While in Ref. [13] a transistor which is narrow in both lateral directions is considered using essentially using the same approximations it is easy to show that (24) also applies to the general case of an electron channel narrow in the z-direction but exhibiting an arbitrary width in the $\mathrm{x}$-direction. 
Scale-invariant formulation In this section we write Eq. 23 with Eq. 24 in a scale-invariant form. To this end in Eq. 25 lengths are normalized to $L$ and energies to $V_{0}$ to obtain

$$
\left[-\frac{1}{\beta} \frac{d^{2}}{d \hat{y}^{2}}+\hat{v}(\hat{y})-\hat{\epsilon}\right] \hat{\psi}(\hat{y}, \hat{\epsilon})=0
$$

where $\beta=2 m^{*} V_{0} L^{2} / \hbar^{2}, \hat{y}=y / L, \hat{\epsilon}=\epsilon / V_{0}, \hat{v}(\hat{y})=V^{\text {eff }}(y) / V_{0}$, and $\hat{\psi}(\hat{y}, \hat{\epsilon})=\psi^{\text {eff }}(y, \epsilon)=$ $\psi^{e f f}\left(L \hat{y}, V_{0} \hat{\epsilon}\right)$. Applying the latter relation to the scattering states in Eq. 26 and 6 yields $\hat{t}^{S}\left(\hat{\epsilon} ; \beta, \hat{v}_{D}\right)=t^{S}(\epsilon)$ so that

$$
\tilde{T}^{e f f}(\epsilon)=\hat{k}_{D}(\hat{\epsilon})\left|\hat{t}^{S}\left(\hat{\epsilon} ; \beta, \hat{v}_{D}\right)\right|^{2}\left[\hat{k}_{S}(\hat{\epsilon})\right]^{-1}=\tilde{T}\left(\hat{\epsilon} ; \beta, \hat{v}_{D}\right)=\tilde{T}\left[\hat{\epsilon} ; l^{2}\left(m-v_{G}\right), \frac{v_{D}}{m-v_{G}}\right] .
$$

For the scaling of the supply function in Eq. 19 we define

$$
s(\hat{\alpha}) \equiv S\left(V_{0} \hat{\alpha}\right)=\frac{1}{2 \sqrt{\pi}} w \sqrt{u} F_{-\frac{1}{2}}\left[\left(v_{G}-m\right) \frac{\hat{\alpha}}{u}\right],
$$

where $\hat{\alpha}=\alpha / V_{0}$. At a given thermal energy the chemical potential $m$ can be calculated from Eq. 14 as

$$
m(u)=u X_{\frac{1}{2}}\left(\frac{4}{3 \sqrt{\pi}} u^{-3 / 2}\right) .
$$

We can now recast Eqs. 23 and Eq. 24 in the dimensionless form given in Eq. 1.

\section{References}

[1] C. Auth, M. Buehler, A. Cappellani, C. Choi, G. Ding et al., Intel Technology Journal 12 (2008), p. 77

[2] M. Ieong, B. Doris, J. Kedzierski, K. Rim, and M. Yang, Science 306 (2004), p. 2057

[3] J. Knoch and J. Appenzeller, Phys. Stat. Sol. (a) 205 (2008), p. 679

[4] J. P. Colinge, Solid State Electronics 48 (2004), p. 897

[5] J. Xiang, W. Lu, Y. Hu, Y. Wu, H. Yan, and C. M. Lieber, Nature 441 (2006), p. 489

[6] J. Knoch, M. Zhang, J. Appenzeller, and S. Mantl, Appl. Phys. A. 87 (2007), p. 351

[7] S. W. Bedell, A. Majumdar, J. A. Ott, J. Arnold and K. Fogel et al., IEEE Electron Dev. Lett. 29 (2008), p. 811

[8] M. K. Hudait, G. Dewey, S. Datta, J. M. Fastenau, and J. Kavalieros et al., IEDM Tech. Dig. (2007), p. 625

[9] M. Yang, V. W. C. Chan, K. K. Chan, L. Shi, D. M. Fried, and J. H. Stathis et al., IEEE Trans. Electron Devices 53 (2006), p. 965

[10] S. M. Sze: Physics of Semiconductor Devices (John Wiley \& Sons, New York, 1981)

[11] S. Thompson, P. Packan, and M. Bohr, Intel Technology Journal Q3 (1998), p. 1 
[12] K. F. Brennan: Introduction to Semiconductor Devices (Cambridge University Press, Cambridge, 2005)

[13] G. A. Nemnes, U. Wulf, and P. N. Racec, J. Appl. Phys. 98 (2005), p. 84308

[14] B. Yu, H. Wang, A. Joshi, Q. Xiang, E. Ibok, and M.-R. Lin, IEDM Tech. Dig., (2001), p. 937

[15] B. Doris, M. Ieong, T. Kanarsky, Y. Zhang, R. A. Roy et al., IEDM Tech. Dig. (2002), p. 267

[16] B. Doyle, R. Arghavani, D. Barlage, S. Datta, M. Doczy et al., Intel Technology Journal 6 (2002), p. 42

[17] S. Tyagi, C. Auth, P. Bai, G. Curello, H. Deshpande et al., IEDM Tech. Dig. (2005), p. 1070

[18] S. Natarajan, M. Armstrong, M. Bost, R. Brain, M. Brazier et al., IEDM Tech. Dig., (2008), p. 1

[19] H. Fukutome, K. Hosaka, K. Kawamura, H. Ohta, Y. Uchino et al., IEEE Electron Dev. Lett. 29 (2008), p. 765

[20] R. H. Dennard, F. H. Gaensslen, H.-N. Yu, V. L. Rideout, E. Bassous, and A. R. LeBlanc, IEEE J. Solid-State Circuits SC-9 (1974), p. 256

[21] J. R. Brews, W. Fichtner, E. H. Nicollian, and S. M. Sze, IEEE Electron Dev. Lett. EDL-1 (1980), p. 221

[22] K. K. Young, IEEE Trans. Electron Devices 36 (1989), p. 504

[23] R-H. Yan, A. Ourmazd, and K. F. Lee, IEEE Trans. Electron Devices 39 (1992), p. 1704

[24] C. P. Auth and J. D. Plummer, IEEE Trans. Electron Devices 18 (1997), p. 74

[25] E. Lind, M. P. Persson, Y-M Niquet, and L.-E. Wernersson, IEEE Trans. Electron Devices 56 (2009), p. 201

[26] S. Datta, Electronic Transport in Mesoscopic Systems (Cambridge University Press, Cambridge, 1995)

[27] A. Svizhenko, M. Anatram, T. R. Govindan, B. Biegel, and R. Venugopal, J. Appl. Phys. 91 (2002), p. 2343

[28] R. Venugopal, Z. Ren, S. Datta, M. S. Lundstrom, and D. Jovanovic, J. Appl. Phys., 92 (2002), p. 3730

[29] D. Mamaluy, D. Vasileska, M. Sabathil, T. Zibold, and P. Vogl, Phys. Rev. B 71 (2005), p. 245321

[30] O. Kurniawan, P. Bai, and E. Li, J. Phys. D 42 (2009), p. 105109

[31] W. R. Frensley, Phys. Rev. B 36 (1987), p. 1570

[32] M. D. Croitoru, V. N. Gladilin, V. M. Fomin, J. T. Devreese, W. Magnus, W. Schoenmaker, and B. Sorée, Solid State Commun., 147 (2008), p. 31

[33] Y. Yamada, H. Tsuchiya, and M. Ogawa, IEEE Trans. Electron Devices 56 (2009), p. 1396

[34] M. Luisier, A. Schenk, W. Fichtner, and G. Klimeck, Phys. Rev. B 74 (2006), p. 205323

[35] K. Majumdar and N. Bhat, J. Appl. Phys. 103 (2008), p. 114503

[36] M. Luisier and A. Schenk, J. Comp. Theor. Nanosci. 5 (2008), p. 1031 
[37] T. B. Boykin, M. Luisier, and G. Klimeck, Phys. Rev. B 77 (2008), p. 165318

[38] D. Querlioz, J. Saint-Martin, K. Huet, A. Bournel, V. Aubry-Fortuna et al, IEEE Trans. Electron Devices 54 (2007), p. 2232

[39] W. Chen, L. F. Register, and S. K. Banerjee, J. Appl. Phys. 103 (2008), p. 24508

[40] K.-M. Liu, W. Chen, L. F. Register, and S. K. Banerjee, J. Appl. Phys. 104 (2008), p. 114515

[41] J. Lusakowski, M. J. Martinez, R. Rengel, T. Gonzales, R. Tauk et al., J. Appl. Phys. 101 (2007), p. 114511

[42] M. V. Fischetti, T. P. O’Regan, S. Narayanan, C. Sachs, S. Jin et al., IEEE Trans. Electron Devices 54 (2007), p. 2116

[43] E. Gnani, S. Reggiani, A. Gnudi, M. Rudan, and G. Baccarani, J. Comp. Theor. Nanosci. 5 (2008), p. 1145

[44] S. Poli, S. Reggiani, A. Gnudi, E. Gnani, and G. Baccarani, IEEE Trans. Electron Devices 55 (2008), p. 313

[45] J. Appenzeller, J. Knoch, M. T. Björk, H. Riel, H. Schmid, W. Riess, IEEE Trans. Electron Devices 55 (2008), p. 2827

[46] M. Lundstrom and J. Guo, Nanoscale Transistors (Springer, Berlin, 2006)

[47] B. Yu, L. Wang, Y. Yuan, P. M. Asbeck, and Y. Taur, IEEE Trans. Electron Devices 55 (2008), p. 2846

[48] K. Natori, IEEE Trans. Electron Devices 55 (2008), p. 2877

[49] A. Khakifirooz, O. M. Nayfeh, and D. Antoniadis, IEEE Trans. Electron Devices 56 (2009), p. 1674

[50] U. Wulf and H. Richter, Solid State Phenomena 156-158 (2010), p. 517

[51] G. A. Nemnes, U. Wulf, and P. N. Racec, J. Appl. Phys. 96 (2004), p. 596

[52] H. Wakabayashi, S. Yamagami, N. Ikezawa, A. Ogura, and M. Narihiro et al., IEDM Tech. Dig. (2003), p. 989

[53] E. Polizzi and N. Ben Abdallah, Phys. Rev. B 66 (2002), p. 245301 